\title{
Homogeneous-Heterogeneous Chemical Reactions of Radiation Hybrid Nanofluid Flow on a Cylinder with Joule Heating: Nanoparticles Shape Impact
}

\author{
Taghreed H. Alarabi ${ }^{1, *}$, Ahmed M. Rashad ${ }^{2}$ (D) and A. Mahdy ${ }^{3}$ \\ 1 Mathematics Department, Faculty of Science, Taibah University, \\ Al-Madinah Al-Munawara P.O. Box 344, Saudi Arabia \\ 2 Mathematics Department, Faculty of Science, Aswan University, Aswan 81528, Egypt; \\ am_rashad@aswu.edu.eg \\ 3 Mathematics Department, Faculty of Science, South Valley University, Qena 82325, Egypt; mahdy@svu.edu.eg \\ * Correspondence: tarabi@taibahu.edu.sa
}

check for updates

Citation: Alarabi, T.H.; Rashad, A.M.; Mahdy, A

Homogeneous-Heterogeneous

Chemical Reactions of Radiation Hybrid Nanofluid Flow on a Cylinder with Joule Heating: Nanoparticles Shape Impact. Coatings 2021, 11, 1490. https://doi.org/10.3390/coatings 11121490

Academic Editor: Rahmat Ellahi

Received: 17 October 2021

Accepted: 1 December 2021

Published: 3 December 2021

Publisher's Note: MDPI stays neutral with regard to jurisdictional claims in published maps and institutional affiliations.

Copyright: (c) 2021 by the authors. Licensee MDPI, Basel, Switzerland. This article is an open access article distributed under the terms and conditions of the Creative Commons Attribution (CC BY) license (https:// creativecommons.org/licenses/by/ $4.0 /)$.

\begin{abstract}
The current analysis aims to exhibit the nanoparticles of $\mathrm{Al}_{2} \mathrm{O}_{3}+\mathrm{Cu}$-water hybrid nanofluid flow for Darcy-Forchheimer with heterogeneous-homogeneous chemical reactions and magnetic field aspects past a stretching or shrinking cylinder with Joule heating. This paper performed not only with the hybrid nanofluid but also the shape of $\mathrm{Al}_{2} \mathrm{O}_{3}$ and $\mathrm{Cu}$ nanoparticles. The model of singlephase hybrid nanofluid due to thermophysical features is utilized for the mathematical formulation. In the present exploration equal diffusions factors for reactants and auto catalyst are instituted. The system of governing equations has been simplified by invoking the similarity transformation. The numerical computations are invoked due to the function bvp4c of Matlab, with high non-linearity. Numerical outcomes illustrated that; sphere shape nanoparticles presented dramatic performance on heat transfer of hybrid nanofluid movement; an opposite behavior is noticed with lamina shape. The local Nusselt number strengthens as the transverse curvature factor becomes larger. In addition, the homogeneous-heterogeneous reactions factors lead to weaken concentration fluctuation.
\end{abstract}

Keywords: hybrid nanofluid; Darcy-Forcheimer; homogeneous-heterogeneous reactions; cylinder; nanoparticles shape; radiation

\section{Introduction}

In recent times, nanofluids have been an active field of research due to its greatly enhanced thermal properties and numerous significant applications in many fields as heat transfer fluids, ferromagnetic fluids, superwetting fluids and detergents, biomedical fluids, polymer nanocomposits, gain media in random lasers, and as building blocks for electronics and optoelectronics devices [1-3]. Choi [4] was the first, who proposed the term "nanofluid". It is obtained by dispersing a small amount of certain nanometer-sized particles (called nanoparticles) of metals, metallic oxides, carbides or carbon nanotubes stably and uniformly in base fluids such as water, ethylene glycol, oil, etc. There are several refrigeration systems based on the absorption phenomenon that are used in many applications such as air conditioning, automobiles [5], solar water distillation [6], freezing of foods [7], etc. Additionally, convection in porous media saturated with nanofluids has extensive experimental and theoretical importance because of its natural occurrence and wide range of applications in many practical situations such as chemical engineering, geothermal energy utilization, oil reservoir modeling, solar energy, building of thermal insulation, nuclear waste disposal, lubrication, biological processes, etc. [8,9]. The experimental study on convective heat transport in nanofluid has been analyzed by Baïri and Laraqi [10] and Torki and Etesami [11]. It is studied theoretically by many researchers for various situations in porous media using Buongiorno's transport model [12]. Many 
of them are Khan and Alzahrani [13], Li et al. [14] and Rawat and Kumar [15]. Ibrahim and Khan [16] examined the influence of viscous dissipation on the mixed convective flow of nanofluids in a porous medium past a stretchable surface. Kumar et al. [17] explored the impacts of thermal radiation on stagnation point polar nanofluid flow over stretchable surface in a porous medium. Loghmani et al. [18] worked on heat transport of nanofluids flow through a porous medium. Khan et al. [19] studied the problem of magneto-nanofluid flow in non-Darcy porous medium.

On other side, hybrid nanoliquids are a new kind of nanofluid. Generally, these kinds of fluid can be made by two separate approaches: (a) dispersion of two or even more nanosized particles to a base fluid, and (b) by suspending the so-called hybrid nanoparticles to a host fluid. The efficacy of hybrid nano-suspensions through their chemo-physical characteristics was examined and proved by several numerical and experimental investigations. The applicability and properties of hybrid nano-suspensions were extensively studied in the literature [19-24]. The magneto-flow and heat transport of hybrid nanoparticles suspended in a non-Newtonian fluid were analyzed by Ghadikolaei et al. [25]. The magneto-natural convection flow of hybrid nanofluid inside a double porous medium was analyzed by Mehryan et al. [26]. Ghadikolaei and Gholinia [27] worked on heat transport and magnetonatural convection flow of hybrid nanofluid over a vertical porous stretchable surface. Suganya et al. [28] evaluated the hybrid nanofluid flow the effect of Darcy-Forchheimer porous medium.

Often, we encounter several processes in nature and industries that also witness mass transport due to variations in concentration. The impact of a chemical reaction is determined by whether it is homogeneous or heterogeneous. The inclusion of pure water and air is impossible in nature. It is possible that any outer matter is naturally there, or that it is combined with air or water. As an outer mass is present in air or liquids, it induces a chemical reaction. Many chemical technologies, such as the manufacture of food processing, glassware or ceramics, and the production of polymers, benefit from the study of related chemical reactions. Lately, many investigations have been published regarding the importance of chemical reaction on hybrid nanofluids flow in porous medium showing their importance in several fields of science and technology; see [29-34].

The main objective of this investigation is to construct a mathematical model $\mathrm{Cu}-\mathrm{Al}_{2} \mathrm{O}_{3}$ / water hybrid nanofluid flow for Darcy-Forchheimer with homogeneous-heterogeneous reactions, non-linear thermal radiation, Joule heating, and heat transfer towards a permeable radially stretching/shrinking cylinder. Five different types of nanoparticle shapes, i.e., sphere, hexahedron, tetrahedron, column and lamina, are taken into account through this contribution. A classical transformation is employed to convert the PDEs into a non-linear system of ODEs.

\section{Materials and Methods}

Consider a steady laminar boundary layer flow of viscous incompressible hybrid nanofluid $\mathrm{Cu}$ and $\mathrm{Al}_{2} \mathrm{O}_{3}$ towards on a stretching/shrinking cylinder with radius $h$ as illustrated in Figure 1. Here, $(x, r)$ is the cylindrical polar coordinates which are assigned in the axial and radial directions, respectively. The velocity of the cylinder is given as $u_{w}(x)=u_{0} x / d$, where constant $u_{0}$ is a characteristic velocity and $d$ is the characteristic length of the cylinder. The nanofluid saturates the given porous medium through DarcyForchheimer relation. The magnetic field of strength $B_{0}$ is imposed normal to the $x$-axis. The interaction of homogeneous and heterogeneous reactions is given as [35]:

$$
A_{1}+2 B_{2} \rightarrow 3 B_{1} \text {, rate }=k_{\mathrm{c}} A B^{2} A_{1} \rightarrow B_{1}, \text { rate }=k_{\mathrm{s}} A
$$

in which the concentration of chemical species $A_{1}$ and $B_{1}$ are symbolized by $A$ and $B$, respectively, while $k_{c}$ and $k_{s}$ denote the constant rates. Using these assumptions together 
with usual boundary layer approximations, the equations of fluid motion can be expressed as $[36,37]$ :

$$
\begin{gathered}
\frac{\partial(r u)}{\partial x}+\frac{\partial(r v)}{\partial r}=0 \\
\rho_{\mathrm{hnf}}\left(u \frac{\partial u}{\partial x}+v \frac{\partial u}{\partial r}\right)=\mu_{\mathrm{hnf}} \frac{1}{r} \frac{\partial}{\partial r}\left(r \frac{\partial u}{\partial r}\right)-\left(\sigma_{\mathrm{hnf}} B_{0}^{2}+\frac{\mu_{\mathrm{hnf}}}{K_{p}}\right) u-\frac{\widetilde{F}}{K_{p}^{1 / 2}} u^{2} \\
\left(\rho c_{p}\right)_{\mathrm{hnf}}\left(u \frac{\partial T}{\partial x} \quad+v \frac{\partial T}{\partial r}\right) \\
=k_{\mathrm{hnf}} \frac{1}{\partial r}\left(r \frac{\partial T}{\partial r}\right)+\sigma_{\mathrm{hnf}} B_{0}^{2} u^{2}-\frac{1}{r} \frac{\partial}{\partial r}\left(r q^{r}\right)+\mu_{\mathrm{hnf}}\left(\frac{\partial u}{\partial r}\right)^{2} \\
+Q_{0}\left(T-T_{\infty}\right) \\
u \frac{\partial A}{\partial x}+v \frac{\partial A}{\partial r}=D_{\mathrm{A}} \frac{1}{r} \frac{\partial}{\partial r}\left(r \frac{\partial A}{\partial r}\right)-k_{c} A B^{2}
\end{gathered}
$$

where $u$ and $v$ denote the fluid velocity in $x$ and $r$ directions, $T$ is the hybrid nanofluid temperature, $D_{\mathrm{A}}$ and $D_{\mathrm{B}}$ are the diffusion coefficient. The term of radiative heat flux is extended by assisting the Rosseland approximation as [38]:

$$
q^{r}=-\frac{4 \sigma^{\star}}{3 k^{\star}} \frac{\partial T^{4}}{\partial r}=-\frac{16 \sigma^{\star}}{3 k^{\star}} T^{3} \frac{\partial T}{\partial r}
$$

here $\sigma^{\star}$ and $k^{\star}$ denote the constant of Stefan-Boltzman and mean absorption factors, respectively.

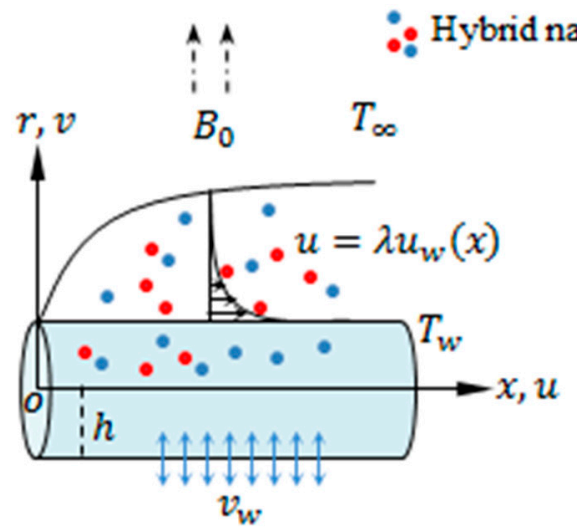

(a) Stretching cylinder $(\lambda>0)$

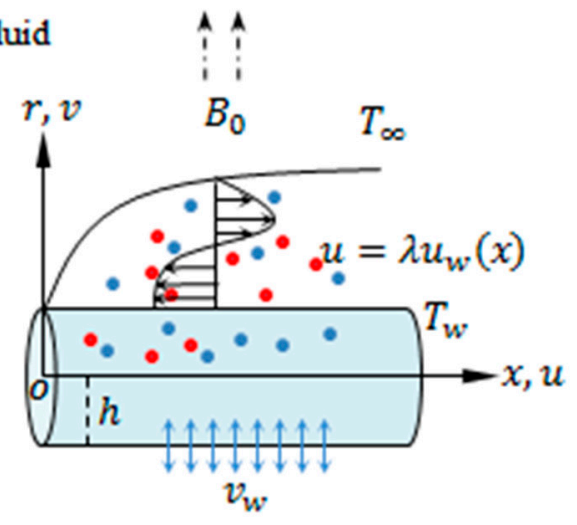

(b) Shrinking cylinder $(\lambda<0)$

Figure 1. Schematic diagram of the flow problem.

The corresponding boundary conditions for a given problem is [38]:

$$
\text { at } r=h,\left\{\begin{array}{l}
u=\lambda u_{w}, v_{w}(r)=-\frac{h}{r}\left(\frac{u_{0} v_{\mathrm{f}}}{d}\right)^{1 / 2} S, T_{w}(x)=T_{\infty}+T_{0}(x / d)^{2} \\
D_{\mathrm{A}} \frac{\partial A}{\partial r}=k_{\mathrm{s}} A, D_{\mathrm{B}} \frac{\partial B}{\partial r}=-k_{\mathrm{s}} A, \\
\quad \text { as } r \rightarrow \infty, u \rightarrow 0, T \rightarrow T_{\infty}, A \rightarrow A_{0}, B \rightarrow 0
\end{array}\right.
$$

Here $v_{w}(r)$ is the constant mass flux velocity through the permeable surface such that $v_{w}>0$ for mass injection and $v_{w}<0$ for mass suction. For the case of an impermeable surface $v_{w}=0$. In addition, the constant stretching/shrinking parameter $\lambda$ signifies the stretching cylinder when $\lambda>0$ and shrinking cylinder when $\lambda<0$. The static cylinder is symbolized by $\lambda=0$.

\subsection{Thermophysical Features}

The modified thermo-physical features regard to the single-phase pattern by Tiwari and Das [39] was imposed early by Devi and Devi $[40,41]$ to exhibit the boundary layer and 
energy equations of hybrid $\mathrm{Cu}-\mathrm{Al}_{2} \mathrm{O}_{3}-\mathrm{H}_{2} \mathrm{O}$ nanofluid around a stretching sheet with variant various factors. Besides, because the boundary layer is analytically formulated, a small number of assumptions were recognized; namely, the main liquid and hybrid nanoparticles were kept in a thermal equilibrium state. The hybrid nanofluid was considered to be stable; thus the impact of hybrid nanoparticles aggregation and sedimentation was ignored. The hybrid nanoparticles are assumed to be spherical, lamina, column, shape.

This portion is devoted to displaying the mathematical expressions of thermophysical features of the hybrid nanofluids and primary fluid which are portrayed in Table 1 due to Gorla et al. [42] and Devi and Devi [40,41]. The physical properties of the base fluid, water, copper $\mathrm{Cu}$ (as first nanoparticle) and alumina $\mathrm{Al}_{2} \mathrm{O}_{3}$ (second nanoparticle) are invoked in Table 2. In addition, the values of the shape factor $m$ are illustrated in Table 3.

Table 1. Thermophysical properties of hybrid nanofluids as Gorla et al. [42].

\begin{tabular}{|c|c|c|}
\hline Properties & Hybrid Nanofluid & Nanofluid \\
\hline Dynamic viscosity & $\mu_{\mathrm{hnf}}=\mu_{\mathrm{bf}}\left(1-\varphi_{2}\right)^{-2.5}$ & $\mu_{\mathrm{bf}}=\mu_{\mathrm{f}}\left(1-\varphi_{1}\right)^{-2.5}$ \\
\hline Density & $\rho_{\mathrm{hnf}}=\left(1-\varphi_{2}\right) \rho_{\mathrm{bf}}+\varphi_{2} \rho_{2}$ & $\rho_{\mathrm{bf}}=\left(1-\varphi_{1}\right) \rho_{\mathrm{f}}+\varphi_{1} \rho_{1}$ \\
\hline Heat capacity & $\left(\rho c_{p}\right)_{\mathrm{hnf}}=\left(1-\varphi_{2}\right)\left(\rho c_{p}\right)_{\mathrm{bf}}+\varphi_{2}\left(\rho c_{p}\right)_{2^{\prime}}$ & $\left(\rho c_{p}\right)_{\mathrm{bf}}=\left(1-\varphi_{1}\right)\left(\rho c_{p}\right)_{\mathrm{f}}+\varphi_{1}\left(\rho c_{p}\right)_{1}$ \\
\hline Thermal conduc. & $\frac{k_{\mathrm{hnf}}}{k_{\mathrm{bf}}}=\frac{\left(k_{2}+(m-1) k_{\mathrm{bf}}\right)-(m-1) \varphi_{2}\left(k_{\mathrm{bf}}-k_{2}\right)}{\left(k_{2}+(m-1) k_{\mathrm{bf}}\right)+\varphi_{2}\left(k_{\mathrm{bf}}-k_{2}\right)}$ & $\frac{k_{\mathrm{bf}}}{k_{\mathrm{f}}}=\frac{\left(k_{1}+(m-1) k_{\mathrm{f}}\right)-(m-1) \varphi_{1}\left(k_{\mathrm{f}}-k_{1}\right)}{\left(k_{1}+(m-1) k_{\mathrm{f}}\right)+\varphi_{1}\left(k_{\mathrm{f}}-k_{1}\right)}$ \\
\hline Electrical conduc. & $\frac{\sigma_{\mathrm{hnf}}}{\sigma_{\mathrm{bf}}}=\left(1+\frac{3\left(\frac{\sigma_{2}}{\sigma_{\mathrm{bf}}}-1\right) \varphi_{2}}{\left(\frac{\sigma_{2}}{\sigma_{\mathrm{bf}}}+2\right)-\left(\frac{\sigma_{2}}{\sigma_{\mathrm{bf}}}-1\right) \varphi_{2}}\right)$, & $\frac{\sigma_{\mathrm{bf}}}{\sigma_{\mathrm{f}}}=\left(1+\frac{3\left(\frac{\sigma_{1}}{\sigma_{\mathrm{f}}}-1\right) \varphi_{1}}{\left(\frac{\sigma_{1}}{\sigma_{\mathrm{f}}}+2\right)-\left(\frac{\sigma_{1}}{\sigma_{\mathrm{f}}}-1\right) \varphi_{1}}\right)$ \\
\hline
\end{tabular}

Table 2. Thermophysical features of base fluid, alumina, and copper [40,42].

\begin{tabular}{ccccc}
\hline Feature & $\left.\rho \mathbf{( k g} / \mathbf{m}^{3}\right)$ & $c_{p}(\mathbf{J} / \mathbf{k g} \cdot \mathbf{K})$ & $k(\mathbf{W} / \mathbf{m} \cdot \mathbf{K})$ & $\sigma(\mathbf{S} / \mathbf{m})$ \\
\hline $\mathrm{H}_{2} \mathrm{O}$ & 997.1 & 4179 & 0.613 & 0.05 \\
$\mathrm{Cu}$ & 8933 & 385 & 401 & $5.96 \times 10^{7}$ \\
Alumina & 3970 & 765 & 40 & $3.69 \times 10^{7}$ \\
\hline
\end{tabular}

Table 3. The empirical shape nanoparticles factor $m$ [43].

\begin{tabular}{cccccc}
\hline Nanoparticles & Sphere & Hexahedron & Tetrahedron & Column & \multicolumn{2}{c}{ Lamina } \\
\hline Shape & 0 & & & & \\
$m$ & 3.0000 & 3.7221 & 4.0613 & 6.3698 & 16.1576 \\
\hline
\end{tabular}

\subsection{Similarity Solution}

Here, we apply the following similarity transformation to convert the considered physical model into non-dimensional mathematical expression:

$$
\begin{gathered}
\eta=\frac{r^{2}-h^{2}}{2 h}\left(\frac{u_{0}}{v_{\mathrm{f}} d}\right)^{1 / 2}, u=\frac{u_{0} x}{d} F^{\prime}(\eta), v=-\frac{h}{r}\left(\frac{u_{0} v_{\mathrm{f}}}{d}\right)^{1 / 2} F(\eta) \\
\theta(\eta)=\frac{T-T_{\infty}}{T_{w}-T_{\infty}}, A=A_{0} N(\eta), B=A_{0} \tilde{N}(\eta)
\end{gathered}
$$

Equation (2) is satisfied automatically and Equations (3)-(6) yield:

$$
\begin{aligned}
(1+2 \alpha \eta) F^{\prime \prime \prime}+ & 2 \alpha F^{\prime \prime}+\frac{\rho_{\mathrm{hnf}}}{\rho_{\mathrm{f}}} \frac{\mu_{\mathrm{f}}}{\mu_{\mathrm{hnf}}}\left(F F^{\prime \prime}-F^{2}\right)-\frac{\sigma_{\mathrm{hnf}}}{\sigma_{\mathrm{f}}} \frac{\mu_{\mathrm{f}}}{\mu_{\mathrm{hnf}}} M_{a} F^{\prime}-\lambda F^{\prime} \\
& -\frac{\mu_{\mathrm{f}}}{\mu_{\mathrm{hnf}}} F^{\star} F^{\prime 2}=0
\end{aligned}
$$




$$
\begin{aligned}
\frac{1}{\operatorname{Pr}}\left(\frac{k_{\mathrm{hnf}}}{k_{\mathrm{f}}}+R_{d}((\right. & \left.N r-1) \theta+1)^{3}\right)\left((1+2 \alpha \eta) \theta^{\prime \prime}+2 \alpha \theta^{\prime}\right)+\frac{\left(\rho c_{p}\right)_{\mathrm{hnf}}}{\left(\rho c_{p}\right)_{\mathrm{f}}}\left(F \theta^{\prime}-2 F^{\prime} \theta\right) \\
+ & \frac{3 R_{d}}{\operatorname{Pr}}(N r-1)(1+2 \alpha \eta)((N r-1) \theta+1)^{2} \theta \prime^{2} \\
& +\frac{\mu_{\mathrm{hnf}}}{\mu_{\mathrm{f}}}(1+2 \alpha \eta) E_{c} F^{\prime \prime 2}+\frac{\sigma_{\mathrm{hnf}}}{\sigma_{\mathrm{f}}} M_{a} E_{c} F^{\prime 2}+Q \theta \\
& (1+2 \alpha \eta) N^{\prime \prime}+2 \alpha N^{\prime}+S c F N^{\prime}-S c K_{c} N \widetilde{N}^{2}=0 \\
\delta & \left((1+2 \alpha \eta) \widetilde{N}^{\prime \prime}+2 \alpha \widetilde{N}^{\prime}\right)+S c F \widetilde{N}^{\prime}+S c K_{c} N \widetilde{N}^{2}=0
\end{aligned}
$$

With the boundary conditions:

$$
\begin{gathered}
F(0)=S, F^{\prime}(0)=\lambda, \theta(0)=1, N^{\prime}(0)=K_{s} N(0), \delta \widetilde{N}^{\prime}(0)=-K_{S} N . \\
F^{\prime}(\infty) \rightarrow 0, \theta(\infty) \rightarrow 0, N(\infty) \rightarrow 1, \widetilde{N}(\infty) \rightarrow 0 .
\end{gathered}
$$

where the obtained parameters are defined as:

$$
\begin{gathered}
\alpha=\left(v_{\mathrm{f}} d /\left(u_{0} h^{2}\right)\right)^{1 / 2} \\
E_{c}=u_{0}^{2} x^{2} /\left(d^{2}\left(c_{p}\right)_{\mathrm{f}}\left(T_{w}-T_{\infty}\right)\right) \\
M_{a}=\sigma_{\mathrm{f}} B_{0}^{2} d /\left(\rho_{\mathrm{f}} u_{0}\right) \\
N r=T_{w} / T_{\infty} \\
R_{d}=16 \sigma^{\star} T_{\infty}^{3} /\left(3 k^{\star} k_{\mathrm{f}}\right) \\
S \\
K_{s}=\left(k_{s} / D_{A}\right) \sqrt{v_{\mathrm{f}} d / u_{0}}
\end{gathered}
$$

stretching or shrinking parameter

rvature factor

$$
\lambda=v_{\mathrm{f}} d /\left(u_{0} K_{p}\right)
$$$$
F^{\star}=\widetilde{F} x /\left(\rho_{\mathrm{f}} K_{p}^{1 / 2}\right)
$$$$
Q=Q_{0} d /\left(\left(\rho c_{p}\right)_{\mathrm{f}} u_{0}\right)
$$$$
S c=v_{\mathrm{f}} / D_{A}
$$$$
\operatorname{Pr}=\mu_{\mathrm{f}}\left(c_{p}\right)_{\mathrm{f}} / k_{\mathrm{f}}
$$$$
\delta=D_{B} / D_{A}
$$
local inertia factor heat generation factor Schmidt number Prandtl number

suction/injection factor

$K_{c}=k_{c} A_{0}^{2} d / u_{0}$ mass diffusion coefficients ratio homogeneous reaction factors

Now, if we put $\delta=1$, i.e., $D_{A}=D_{B}$, thus $N(\eta)+\widetilde{N}(\eta)=1$.

Again, Equations (11) and (12) yield:

$$
(1+2 \alpha \eta) N^{\prime \prime}+2 \alpha N^{\prime}+S c F N^{\prime}-S c K_{c} N(1-N)^{2}=0
$$

with, the corresponding boundary condition

$$
N^{\prime}(0)=K_{s} N(0), N(\infty) \rightarrow 1
$$

The skin friction coefficient $C_{f}$ and the Nusselt number $N u$, are defined as:

$$
C_{f}=\frac{\mu_{\mathrm{hnf}}}{\rho_{\mathrm{f}} u_{w}^{2}}\left(\frac{\partial u}{\partial r}\right)_{r=h}, N u=\frac{x q_{w}}{k_{\mathrm{f}}\left(T_{w}-T_{\infty}\right)}
$$

where:

$$
q_{w}=\left(-k_{\mathrm{hnf}} \frac{\partial T}{\partial r}+q^{r}\right)_{r=h}
$$

Substituting Equation (8) into Equation (16), we get the following formula for skinfriction factor and Nusselt number,

$$
\operatorname{Re}^{\frac{1}{2}} C_{f}=\frac{\mu_{\mathrm{hnf}}}{\mu_{\mathrm{f}}} F^{\prime \prime}(0), R e^{-1 / 2} N u=-\left(\frac{k_{\mathrm{hnf}}}{k_{\mathrm{f}}}+R_{d}((N r-1) \theta(0)+1)^{3}\right) \theta \prime(0)
$$

where $R e=\frac{x u_{w v}}{v_{f}}$ shows the local Reynolds number, $f^{\prime \prime}(0), \theta^{\prime}(0)$ indicate the velocity and temperature gradients.

\section{Results}

The salient parameters of the flow and heat transfer behavior are exhibited for the hydromagnetic hybrid $\mathrm{Al}_{2} \mathrm{O}_{3}-\mathrm{Cu}$ nanofluid flow and heat transfer for Darcy-Forchheimer with homogeneous-heterogeneous reactions by a permeable stretching or shrinking cylinder. The exhibited non-linear flow differential Equations (9), (10) and (14) with the four 
proper boundary conditions expressed in Equations (13) and (15) are a strenuous way to obtain a solution. Hence, these flow equations are solved numerically, due to the function bvp4c from MATLAB R2008b for representative values of the dimensionless factors. The imposed Matlab code, bvp4c, is developed implementing a FDM that use the three-stage Lobatto IIIa expression. This is a collocation method with fourth-order accuracy. Through this technique, the ODEs. (9), (10) and (14) are first converted to a system of first order by presenting new transformations, i.e., initial value problem (IVP). The mesh select and error control are related to the residual of the continuous solution. Comprehensive outcomes were performed for miscellaneous values of the factors describing fluid motion. Validation of numerical computations is achieved by comparing the outcomes of our investigation with Khashi'ie et al. [36] and Waini et al. [44], which are displayed in Table 4 for various values of $\varphi_{\mathrm{Cu}}$, when $\varphi_{\mathrm{Al}_{2} \mathrm{O}_{3}}=0.1, M_{a}=S=\alpha=E_{c}=R_{d}=0, \lambda=1$ and $\operatorname{Pr}=6.135$. From the presented data it is noticed that the calculated outcomes are completely in agreement with previous published computations. The physical parameters were fixed as $m=6.3698$, i.e., column nanoparticle shape, $E_{c}=0.1, M_{a}=0.5, R_{d}=0.2, N r=0.1, \alpha=1, S=1$, $\lambda=1, S c=0.62, Q=0.2, F^{\star}=0.4, K_{c}=K_{s}=0.5, \varphi_{\mathrm{Al}_{2} \mathrm{O}_{3}}=\varphi_{\mathrm{Cu}}=0.03$. Figure 2 illustrates the impact of miscellaneous nanoparticles shapes of the hybrid nanofluid through the boundary layer flow of $\mathrm{Cu}+\mathrm{Al}_{2} \mathrm{O}_{3}-\mathrm{H}_{2} \mathrm{O}$, at a fixed volume fraction $\varphi_{\mathrm{Al}_{2} \mathrm{O}_{3}}=\varphi_{\mathrm{Cu}}=0.03$.

Table 4. Comparison of our results for skin friction.

\begin{tabular}{cccc}
\hline$\varphi_{\boldsymbol{C} \boldsymbol{u}}$ & Khashi'ie et al. [36] & Waini et al. [44] & Present Study \\
\hline 0.005 & -1.327097962 & -1.327098 & -1.327630 \\
0.02 & -1.40949019 & -1.409490 & -1.406323 \\
0.04 & -1.520721211 & -1.520721 & -1.512846 \\
0.06 & -1.634118687 & -1.634119 & -1.621717 \\
\hline
\end{tabular}
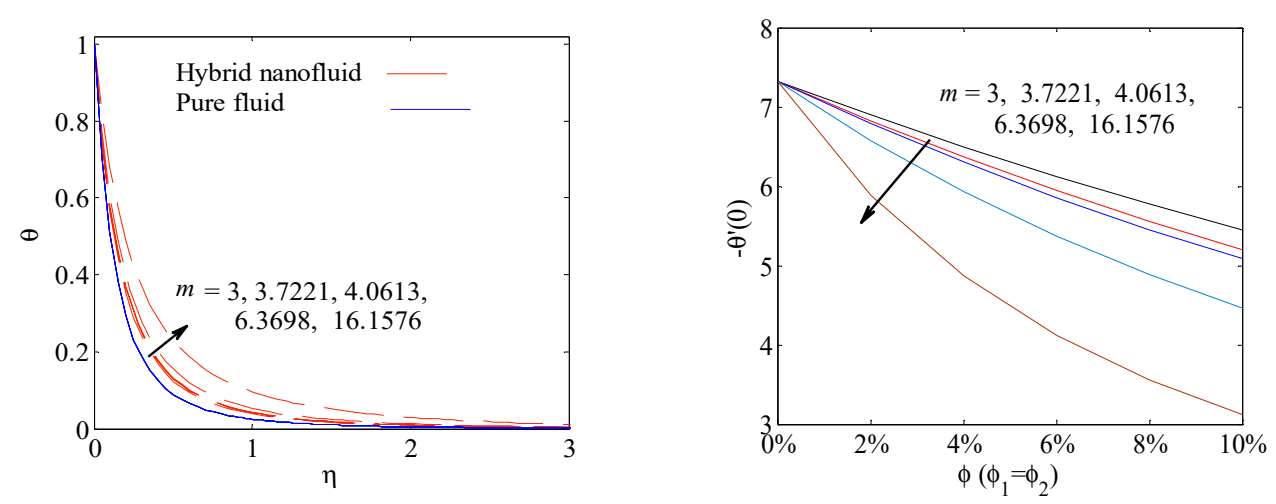

Figure 2. Temperature distribution and Nusselt number for variant values of shape of nanoparticles.

Due to the graph, it is clarified that the dimensionless temperature of the hybrid nanofluid boosts as the values of factor $m$ enhances. Spherical shape nanoparticles give the minimum temperature fluctuations, followed by hexahedron, tetrahedron, column, and lamina. This can be explained as the sphere shape nanoparticles have lowest thermal conductivity and viscosity of those lamina shape nanoparticles. The spherical shaped nanoparticle, due to its improved surface area, leads to dragging more heat from the boundary layer, whereas this impact is less evident for the other shapes. This accounts for the maximum rate of heat transfer at the boundary for the spherical-shaped nanoparticles as can be perceived in Figure 2 and Table 5.

Figure 3 obviously presents the hybrid nanofluid velocity fluctuations $F^{\prime}(\eta)$ which start from maximum value at the surface of the cylinder and then weaken until they attain the lowest value of the boundary layer for miscellaneous positive values of magnetic field factor $M_{a}(=0,0.1,0.5,1$ and 2$)$. This figure belays that the velocity curves minimize with higher values of $M_{a}$. In addition, the skin friction coefficient strengthens as $M_{a}$ enhances. 
An opposite influence is clear for temperature curves Figure 4, i.e., the hybrid nanofluid temperature fluctuations boost with the increase in magnetic field factor.

The rate of heat transfer variations for miscellaneous values of $M_{a}, M_{a}$ yields the Nusselt number to weaken as shown. This is reality because of the boosting intensity of the magnetic field that outputs resistive force, i.e., an opposite force to the trend of fluid movement, that is named the "Lorentz force". The created force has the susceptibility to minimize the velocity boundary layer and strengthen the thermal boundary-layer thickness.

Table 5. Velocity and temperature gradient as $\varphi_{\mathrm{Cu}}=\varphi_{\mathrm{Al}_{2} \mathrm{O}_{3}}=0.03$.

\begin{tabular}{cccccc}
\hline$M_{a}$ & $m$ & $E_{c}$ & $\alpha$ & $-\boldsymbol{F}^{\prime \prime}(0)$ & $-\boldsymbol{\theta}^{\prime}(0)$ \\
\hline 0.0 & 3.0 & 0.1 & 1.0 & 2.567727 & 6.276612 \\
0.1 & - & - & - & 2.603955 & 6.243702 \\
0.5 & - & - & - & 2.741170 & 6.119466 \\
1.0 & - & - & - & 2.898269 & 5.978082 \\
0.5 & 3.0 & - & - & 2.741170 & 6.119466 \\
- & 3.7221 & - & - & 2.741170 & 5.938033 \\
- & 4.0613 & - & - & 2.741170 & 5.857272 \\
- & 6.3698 & - & - & 2.741173 & 5.373093 \\
- & 3.0 & 0.0 & - & 2.741168 & 6.917546 \\
- & - & 0.1 & - & 2.741170 & 6.119466 \\
- & - & 0.3 & - & 2.741172 & 4.000560 \\
- & - & 0.5 & - & 2.741176 & 2.931343 \\
- & - & 0.1 & 0.0 & 2.356231 & 6.081831 \\
- & - & - & 0.1 & 2.395392 & 6.085194 \\
- & - & - & 0.5 & 2.550767 & 6.097921
\end{tabular}
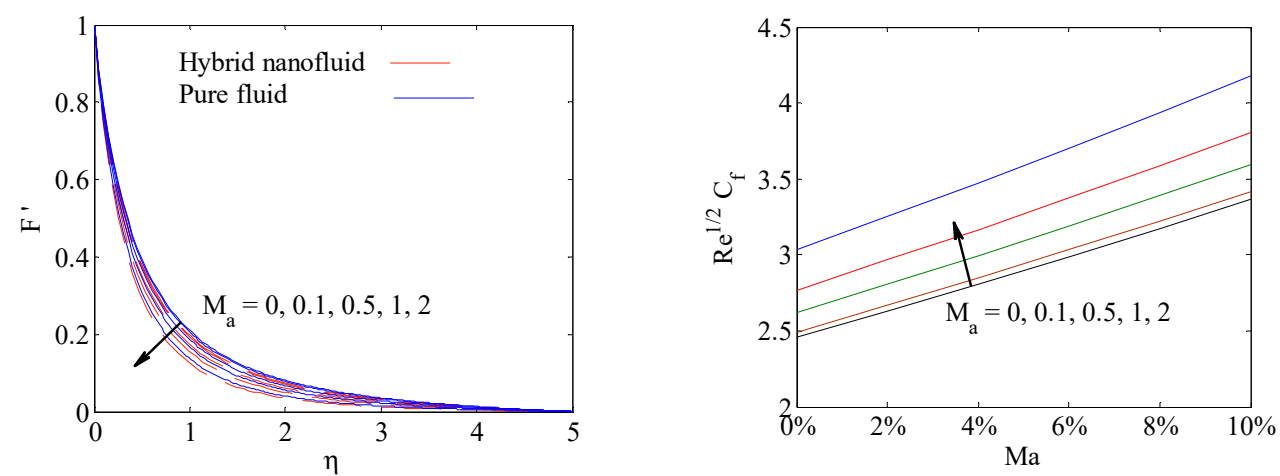

Figure 3. Velocity distribution and skin friction coefficient for variant values of $M_{a}$ parameter.
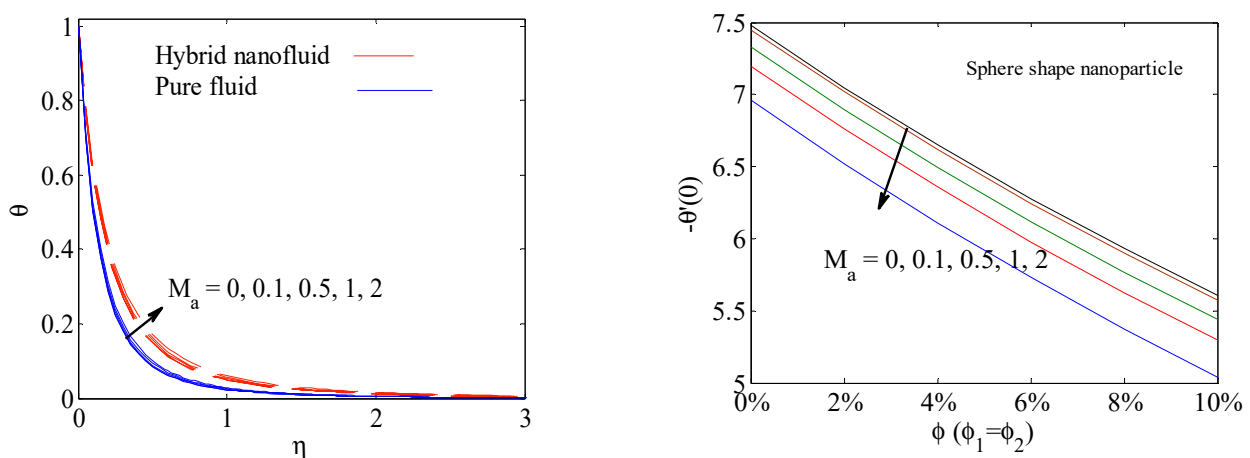

Figure 4. Temperature distribution and Nusselt number for variant values of $M_{a}$ parameter.

The impact of suction/injection $S$ factor on non-dimensional hybrid nanofluid velocity and temperature profiles is plotted in Figure 5. An increment in suction/injection factor has 
the susceptibility to force the fluid moves into an unoccupied space that creates changes in the boundary layer. Therefore, the hybrid nanofluid velocity and temperature are dawdled for enhancing the $S$ factor. Due to forcing the liquid through a permeable cylinder, it is clear that the hybrid nanofluid temperature weakens due to enlarging the $S$ parameter. The influence of shrinking $\lambda<0$ or stretching $\lambda>0$ factor has been presented in Figure 6 . For stretching cases with improving $\lambda$ the hybrid nanofluid velocity curves boost but the temperature reduces. The opposite behavior is seen in the case of shrinking, i.e., with increasing $\lambda$ the temperature enhances and the velocity reduces.
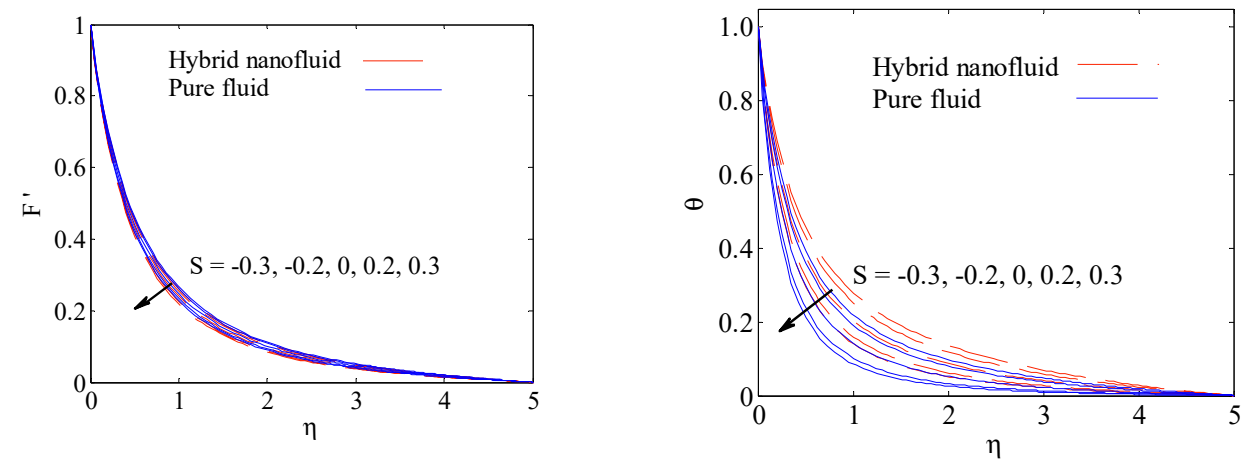

Figure 5. Velocity and temperature distributions for variant values of $S$ parameter.
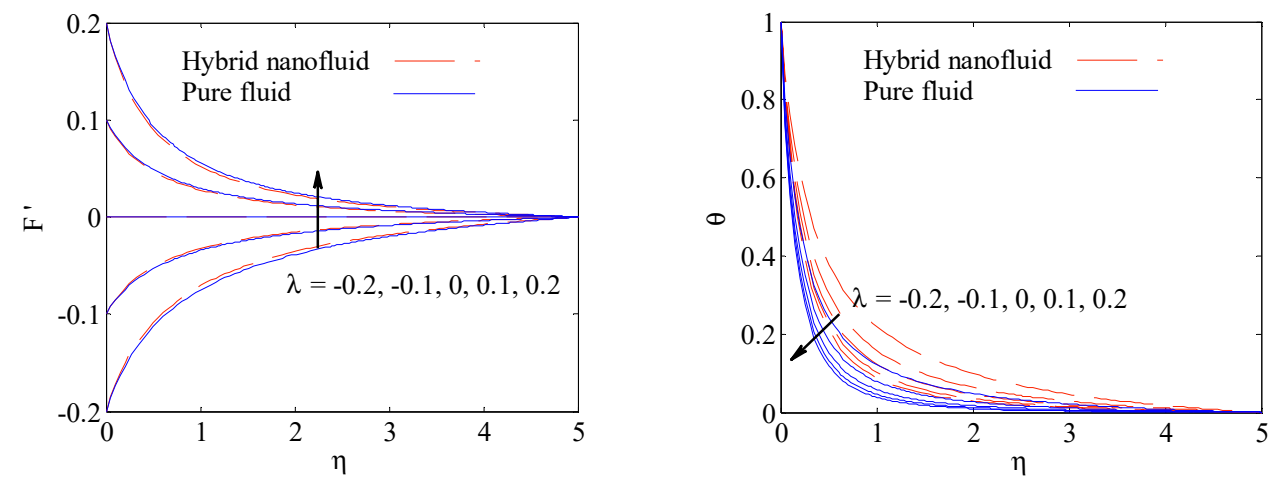

Figure 6. Velocity and temperature distributions for variant values of $\lambda$ parameter.

The aspects of the curvature factor $\alpha$ on $F^{\prime}(\eta)$ and $\theta(\eta)$ fluctuations are presented in Figure 7. From this figure, we perceive that the velocity of the hybrid nanofluid has a clear relation to the curvature factor $\alpha$. This is due to $\alpha$ possessing a reverse relation with the curvature radius, so the contact horizontal region of the cylinder reduces with limited impedance to the hybrid nanofluid flow generated. Again, an enhancement in $\alpha$ lead to boost both in velocity and temperature distributions; physically, kinetic energy boosts with higher values of curvature factor because of the strengthening $\theta(\eta)$ profile of hybrid nanofluid. The influence of viscous dissipation factor (Eckert number) $E_{c}$ on hybrid nanofluid temperature and rate of heat transfer is invoked in Figure 8. The thermal boundary-layer thickness strengthens for increasing values of $E_{c}$, whilst the gradient of hybrid nanofluid temperature at cylinder surface that formulated in terms of local Nusselt number weakens. This behavior can be interpreted as the viscous dissipation boosts thus the thermal conductivity of the flow uplifts, in which produces to strengthen the momentum and thermal boundary layers. 

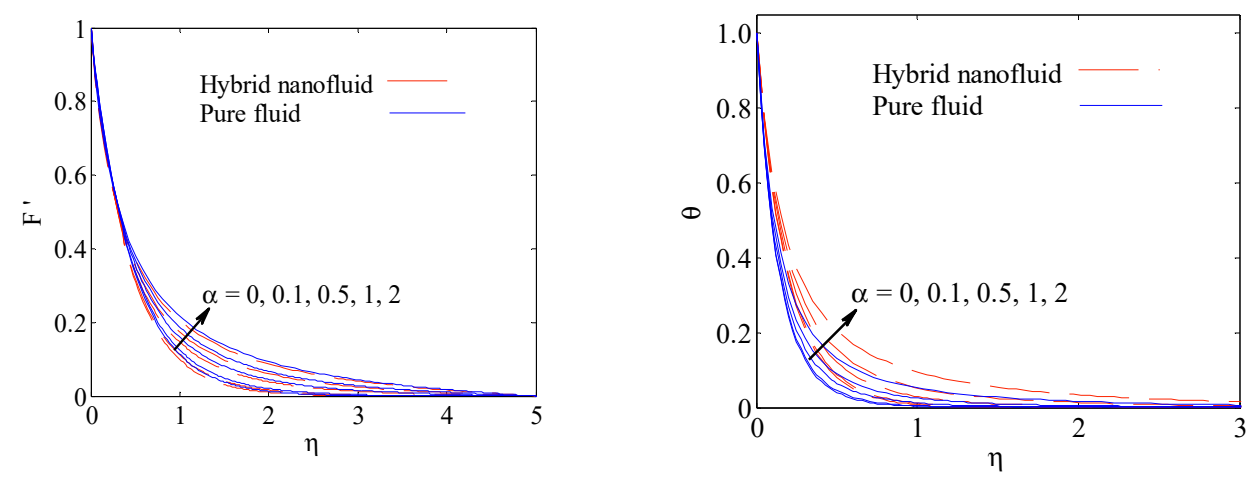

Figure 7. Velocity and temperature distributions for variant values of $\alpha$ parameter.
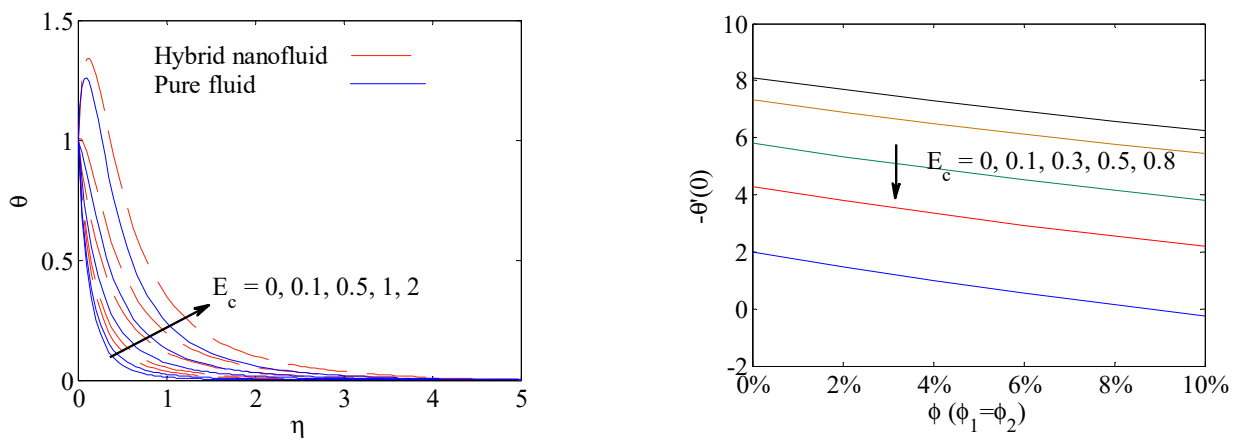

Figure 8. Temperature distribution and Nusselt number for variant values of $E_{\mathcal{c}}$ parameter.

Figure 9 reveals the impact of radiation $R_{d}$ and $N r$ factors on hybrid nanofluid temperature curves. As illustrated, an increment in radiation factor results in boosts in thermal boundary layer thickness. The same behavior is gained due to the surface temperature factor $N r$, that is the curves of hybrid nanofluid temperature enhance with increasing $N r$. An increment in thermal radiation provides energy to the particles of the hybrid nanofluid which invokes an augment in both temperature and thermal boundary-layer thickness. Basically, this factor becomes obvious at the surface and is so helpful to strengthen the temperature distributions.
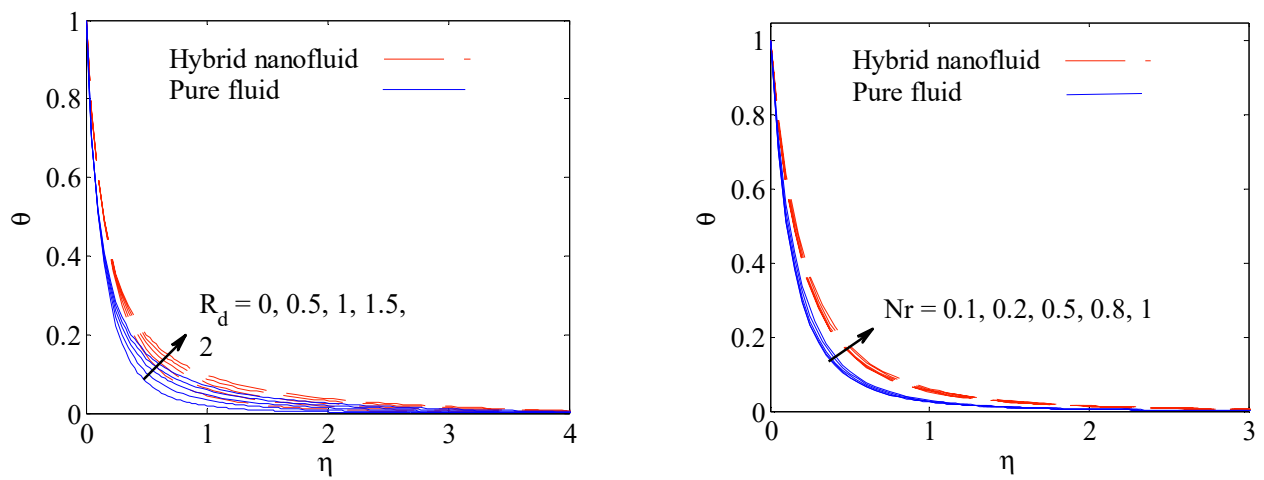

Figure 9. Temperature distribution for variant values of $R_{d}$ and $N r$ parameters.

Aspects of the dimensionless intensity of homogeneous reaction factor $K_{c}$, heterogeneous reaction factor strength $K_{s}$ on non-dimensional concentration fluctuations are highlighted in Figure 10. Due to an increment in values of homogeneous reaction strength $K_{c}$ and heterogeneous reaction intensity $K_{s}$, a diminution in the concentration fluctuation $N(\eta)$ is noticed, since in homogeneous reaction the reactants are wasted, and after certain value of the similarity variable $\eta$ this influence vanishes. In addition, the Schmidt number 
Sc stands for the ratio of momentum to mass diffusivity. Thus, by enhancing Schmidt number, minimum mass diffusivity replaced yielding hybrid nanofluid concentration to weaken as depicted in Figure 11. The curvature factor $\alpha$ has the same aspect of the concentration curves, i.e., $\alpha$ leads to reducing the hybrid nanofluid concentration fluctuations, Figure 11.

Variations of skin friction coefficient, rate of heat transfer and gradient concentration at the surface of cylinder for miscellaneous parameters have been portrayed in Tables 5-7 for sphere shape nanoparticles. As given, the parameters $M_{a}, m, E_{c}, Q, N r$ and $F^{\star}$ lead to weakening the temperature gradient at the surface, whilst the $\alpha$ and $R_{d}$ yield enhance it. The skin friction coefficient improves with escalating $M_{a}, F^{\star}$ and $\alpha$. In addition, the gradient of concentration strengthens as $S c$ and $K_{s}$ escalate and it reduces with increasing $K_{c}$.
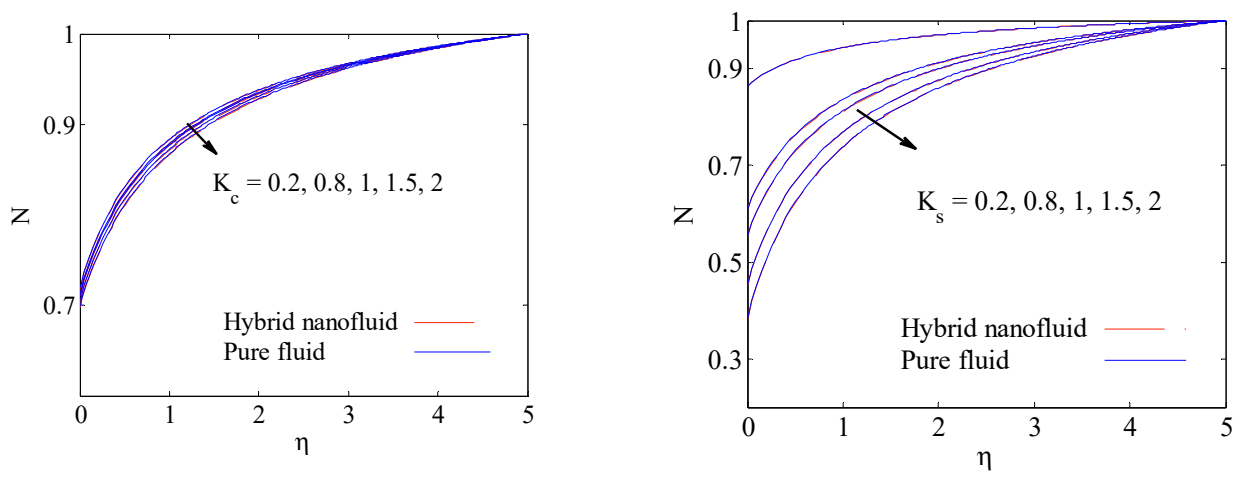

Figure 10. Concentration distribution for variant values of $K_{c}$ and $K_{s}$ parameters.
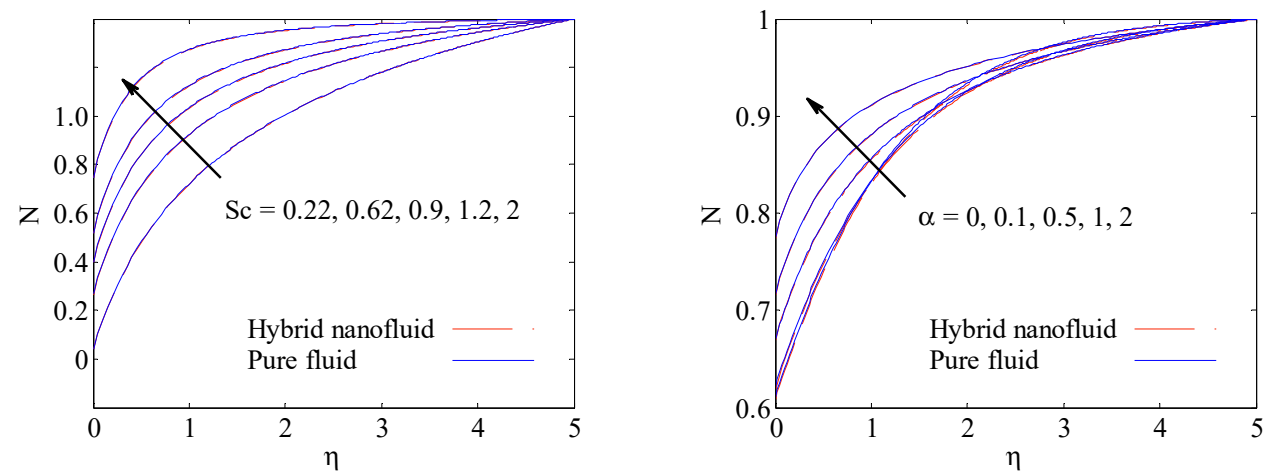

Figure 11. Concentration distribution for variant values of $S c$ and $\alpha$ parameters.

Table 6. Velocity and temperature gradient as $\varphi_{\mathrm{Cu}}=\varphi_{\mathrm{Al}_{2} \mathrm{O}_{3}}=0.03$.

\begin{tabular}{|c|c|c|c|c|c|}
\hline$Q$ & $N r$ & $R_{d}$ & $F^{\star}$ & $F^{\prime \prime}(0)$ & $-\theta^{\prime}(0)$ \\
\hline 0.0 & 0.1 & 0.2 & 0.4 & 2.741159 & 6.308612 \\
\hline 0.2 & - & - & - & 2.741170 & 6.119466 \\
\hline 0.5 & - & - & - & 2.741163 & 5.791617 \\
\hline 1.0 & - & - & - & 2.741173 & 4.647444 \\
\hline 0.2 & 0.1 & - & - & 2.741170 & 6.119466 \\
\hline - & 0.2 & - & - & 2.741170 & 6.118418 \\
\hline- & 0.5 & - & - & 2.741170 & 6.030476 \\
\hline- & 1.0 & - & - & 2.741173 & 5.365267 \\
\hline- & 0.1 & 0.0 & - & 2.741170 & 6.087991 \\
\hline - & - & 0.4 & - & 2.741168 & 6.150117 \\
\hline- & - & 0.8 & - & 2.741168 & 6.210376 \\
\hline- & - & 1.2 & - & 2.741168 & 6.270762 \\
\hline- & - & 0.2 & 0.0 & 2.682743 & 6.140906 \\
\hline- & - & - & 0.5 & 2.755528 & 6.114194 \\
\hline- & - & - & 0.9 & 2.812024 & 6.093441 \\
\hline- & - & - & 1.0 & 2.853459 & 6.078212 \\
\hline
\end{tabular}


Table 7. Concentration gradient as $\varphi_{\mathrm{Cu}}=\varphi_{\mathrm{Al}_{2} \mathrm{O}_{3}}=0.03$.

\begin{tabular}{cccc}
\hline$S c$ & $\boldsymbol{K}_{\boldsymbol{c}}$ & $\boldsymbol{K}_{\boldsymbol{s}}$ & $\boldsymbol{N}^{\prime}(\mathbf{0})$ \\
\hline 0.22 & 0.5 & 0.5 & 0.329924 \\
0.62 & - & - & 0.358027 \\
0.90 & - & - & 0.374613 \\
1.2 & - & - & 0.389593 \\
0.62 & 0.2 & - & 0.359505 \\
- & 0.8 & - & 0.356460 \\
- & 1.0 & - & 0.355361 \\
- & 1.5 & - & 0.352399 \\
- & 0.5 & 0.2 & 0.172884 \\
- & - & 0.8 & 0.488269 \\
- & - & 1.0 & 0.555509 \\
- & - & 1.5 & 0.680357 \\
\hline
\end{tabular}

\section{Conclusions}

This contribution addresses the $\mathrm{Cu}+\mathrm{Al}_{2} \mathrm{O}_{3}$ nanoparticles shape impact on MHD hybrid nanofluid flow and heat transfer past stretching and shrinking a horizontal permeable cylinder with isothermal heterogeneous and homogeneous reactions. In this regard, the striking attention of the present analysis is illustrated as follows

1. Factors $K_{s}$ and $K_{c}$ have similar impacts on concentration fluctuations. Higher values of $K_{c}$ and $K_{s}$ factors lead to weakened concentration curves.

2. Eckert number and magnetic field strengthen $\theta(\eta)$ distributions but weakens the local Nusselt number.

3. Sphere shape nanoparticles presented a dramatic performance on heat transfer of hybrid nanofluid movement whereas an opposite characteristic is noticed with lamina shape.

4. The maximum $\theta(\eta)$ is generated by the lamina-shaped nanoparticles and the minimum value is obtained by sphere shape nanoparticles, but column shape presented medium execution on heat transfer.

Author Contributions: T.H.A., formal analyses, original draft, investigation, writing; A.M.R., investigation, supervision, writing-review, conceptualization, data curation; A.M., visualization, validation. All authors have read and agreed to the published version of the manuscript.

Funding: This research received no external funding.

Institutional Review Board Statement: Not applicable.

Informed Consent Statement: Not applicable.

Data Availability Statement: Data is contained within the article.

Conflicts of Interest: The authors declare no conflict of interest.

$\begin{array}{ll}\begin{array}{l}\text { Abbreviations } \\ \text { Nomenclature }\end{array} & \\ A, B & \text { Concentrations of chemical species } \\ \mathrm{B}_{0} & \text { Strength of magnetic field } \\ C_{f} & \text { Skin friction coefficient } \\ c_{p} & \text { Specific heat } \\ D_{A}, D_{B} & \text { Diffusion coefficients of A, B species } \\ \mathrm{Ec} & \text { Eckert number } \\ F & \text { Non-dimensional stream function } \\ F^{*} & \text { Local inertia factor } \\ h & \text { Radius of cylinder }\end{array}$




$\begin{array}{ll}k & \text { Thermal conductivity } \\ k^{*} & \text { Mean absorption parameter } \\ K_{c} & \text { Homogeneous reaction strength } \\ K_{s} & \text { Heterogeneous reaction strength } \\ m & \text { Nanoparticle Empirical shape factor } \\ \mathrm{M}_{a} & \text { Magnetic field parameter } \\ \mathrm{Nr} & \text { Surface temperature excess } \\ \mathrm{Nu} & \text { Nusselt number } \\ \mathrm{Pr} & \text { Prandtl number } \\ \mathrm{Q} & \text { Heat generation coefficient } \\ \mathrm{q}^{r} & \text { Radiative heat flux } \\ \mathrm{R}_{d} & \text { Radiation parameter } \\ \mathrm{Re} & \text { Reynolds number } \\ \mathrm{S} & \text { Mass transpiration parameter } \\ \mathrm{Sc} & \text { Schmidt number } \\ T & \text { Dimensional temperature } \\ (u, v) & \text { Velocity components } \\ v_{w} & \text { Constant mass flux, ms } \\ (x, r) & \text { Cylindrical polar coordinates } \\ \mathrm{Greek} \text { Symbols } & \\ \mu & \text { Dynamic viscosity } \\ \rho & \text { Fluid density } \\ \psi & \text { Stream function } \\ \varphi & \text { Nanoparticles } \\ \lambda & \text { Stretching/shrinking parameter } \\ \theta & \text { Dimensionless temperature } \\ v & \text { Kinematic viscosity } \\ \eta & \text { Curvature parameter } \\ \alpha & \text { Stectrical conductivity } \\ \sigma & \\ \sigma * & \text { Conditions at the surface } \\ \mathrm{Subscripts} & \\ w & \mathrm{f}, \mathrm{hnf}\end{array}$

\section{References}

1. Das, S.K.; Choi, S.U.; Yu, W.; Pradeep, T. Nanofluids: Science and Technology; John Wiley and Sons, Inc.: Hoboken, NJ, USA, 2000.

2. Wang, L.; Quintard, M. Nanofluids of the Future. In Advances in Transport Phenomena, 1st ed.; Springer: Berlin/Heidelberg, Germany, 2009; p. 179.

3. Puliti, G.; Paolucci, S.; Sen, M. Nanofluids and their properties. Appl. Mech. Rev. 2011, 64, 30803.

4. Choi, S.; Eastman, J.A. Enhancing thermal conductivity of fluids with nanoparticles. In Proceedings of the ASME International Mechanical Congress and Exposition, San Francisco, CA, USA, 12-17 November 1995.

5. Ramanathan, A.; Gunasekaran, P. Simulation of absorption refrigeration system for automobile application. Therm. Sci. 2008, 12, 5. [CrossRef]

6. Kuznetsov, I.A.; Greenfield, M.J.; Mehta, Y.U.; Merchan, W.; Salkar, G.; Saveliev, A.V. Increasing the solar cell power output by coating with transition metal oxide nanorods. Appl. Energy 2011, 88, 4218. [CrossRef]

7. Singh, R.P.; Heldman, D.R. Introduction to Food Engineering, 5th ed.; Academic Press: San Diego, CA, USA, 2014.

8. Nield, D.A.; Bejan, A. Convection in Porous Media, 5th ed.; Springer Nature: Cham, Switzerland, 2017.

9. Khanafer, K.; Vafai, K. Application of nanofluids in porous medium: A critical Review. J. Therm. Anal. Calorim. 2019, 135, 1479. [CrossRef]

10. Baïri, A.; Laraqi, N. Experimental quantification of natural convective heat transfer within annulus space filled with a $\mathrm{H}_{2} \mathrm{O}-\mathrm{Cu}$ nanofluid saturated porous medium. Application to electronics cooling. Exp. Heat Transf. 2019, 32, 364-375. [CrossRef]

11. Torki, M.; Etesami, N. Experimental investigation of natural convection heat transfer of $\mathrm{SiO}_{2} /$ water nanofluid inside inclined enclosure. J. Therm. Anal. Calorim. 2020, 139, 1565-1574. [CrossRef]

12. Buongiorno, J. Convective transport in nanofluids. J. Heat Mass Transf. 2005, 128, 240. [CrossRef]

13. Khan, M.I.; Alzahrani, F. Free convection and radiation effects in nanofluid (Silicon dioxide and Molybdenum disulfide) with second order velocity slip, entropy generation, Darcy-Forchheimer porous medium. Int. J. Hydrog. Energy 2021, 46, 1362-1369. [CrossRef] 
14. Li, Y.X.; Alqsair, U.F.; Ramesh, K.; Khan, S.U.; Khan, M.I. Nonlinear heat source/sink and activation energy assessment in double diffusion flow of micropolar (non-newtonian) nanofluid with convective conditions. Arab. J. Sci. Eng. 2021. [CrossRef]

15. Rawat, S.K.; Kumar, M. Cattaneo-Christov heat flux model in flow of copper water nanofluid through a stretching/shrinking sheet on stagnation point in presence of heat generation/absorption and activation energy. Int. J. Appl. Comput. Math. 2020, 6. [CrossRef]

16. Ibrahim, M.; Khan, M.I. Mathematical modeling and analysis of SWCNT-Water and MWCNT-Water flow over a stretchable sheet. Comput. Methods Prog. Biomed. 2020, 187, 105222. [CrossRef] [PubMed]

17. Kumar, B.; Seth, G.S.; Nandkeolyar, R. Regression model and successive linearization approach to analyse stagnation point micropolar nanofluid flow over a stretching sheet in a porous medium with nonlinear thermal radiation. Phys. Scr. 2019, 94, 115211. [CrossRef]

18. Loghmani, G.B.; Heydari, M.; Hosseini, E.; Rashidi, M.M. A numerical simulation of MHD flow and radiation heat transfer of nanofluids through a porous medium with variable surface heat flux and chemical reaction. J. Math. Ext. 2019, 13, 31-67.

19. Khan, M.I.; Alzahrani, F.; Hobiny, A. Simulation and modeling of second order velocity slip flow of micropolar ferrofluid with Darcy-Forchheimer porous medium. J. Mater. Res. Technol. 2020, 9, 7335-7340. [CrossRef]

20. Sarkar, J.; Ghosh, P.; Adil, A. A review on hybrid nanofluids: Recent research, development and applications. Renew. Sustain. Energy Rev. 2015, 43, 164-177. [CrossRef]

21. Sidik, N.A.C.; Adamu, I.M.; Jamil, M.M.; Kefayati, G.H.R.; Mamat, R.; Najafi, G. Recent progress on hybrid nanofluids in heat transfer applications: A comprehensive review. Int. Commun. Heat Mass Transf. 2016, 78, 68-79. [CrossRef]

22. Sundar, L.S.; Sharma, K.V.; Singh, M.K.; Sousa, A.C.M. Hybrid nanofluids preparation, thermal properties, heat transfer and friction factor-A review. Renew. Sustain. Energy Rev. 2017, 68, 185-198. [CrossRef]

23. Babu, J.R.; Kumar, K.K.; Rao, S.S. State-of-art review on hybrid nanofluids. Renew. Sustain. Energy Rev. 2017, 77, 551-565. [CrossRef]

24. Huminic, G.; Huminic, A. Hybrid nanofluids for heat transfer applications-A state-of-the-art review. Int. J. Heat Mass Transf. 2018, 125, 82-103. [CrossRef]

25. Ghadikolaei, S.S.; Hosseinzadeh, K.; Hatami, M.; Ganji, D.D. MHD boundary layer analysis for micropolar dusty fluid containing hybrid nanoparticles over a porous medium. J. Mol. Liq. 2018, 268, 813-823. [CrossRef]

26. Mehryan, S.A.M.; Sheremet, M.A.; Soltani, M.; Izadi, M. Natural convection of magnetic hybrid nanofluid inside a double porous medium using two-equation energy model. J. Mol. Liq. 2019, 277, 959-970. [CrossRef]

27. Ghadikolaei, S.S.; Gholinia, M. Terrific effect of $\mathrm{H}_{2}$ on $3 \mathrm{D}$ free convection MHD flow of $\mathrm{C}_{2} \mathrm{H}_{6} \mathrm{O}_{2}-\mathrm{H}_{2} \mathrm{O}$ hybrid base fluid to dissolve $\mathrm{Cu}$ nanoparticles in a porous space considering the thermal radiation and nanoparticle shapes effects. Int. J. Hydrog. Energy 2019, 44, 17072-17083. [CrossRef]

28. Suganya, S.; Muthtamilselvan, M.; Al-Amri, F.; Abdalla, B.; Doh, D.H. Filtration of radiating and reacting SWCNTMWCNT/Water hybrid flow with thesignificance of Darcy-Forchheimer porous medium. Arab. J. Sci. Eng. 2021, 46, 1981-1995. [CrossRef]

29. Zainal, N.A.; Nazar, R.; Naganthran, K.; Pop, I. Flow and heat transfer over a permeable moving wedge in a hybrid nanofluid with activation energy and binary chemical reaction. Int. J. Numer. Methods Heat Fluid Flow 2021. [CrossRef]

30. Waini, I.; Ishak, A.; Pop, I. Hybrid nanofluid flow with homogeneous-heterogeneous reactions. CMC-Comput. Mater. Contin. 2021, 68. [CrossRef]

31. Ahmad, S.; Nadeem, S. Analysis of activation energy and its impact on hybrid nanofuid in the presence of Hall and ion slip currents. Appl. Nanosci. 2020, 10, 5315-5330. [CrossRef]

32. Waqas, H.; Farooq, U.; Bukhari, F.F.; Alghamdi, M.; Muhammad, T. Chemically reactive transport of magnetized hybrid nanofluids through Darcian porous medium. Case Stud. Therm. Eng. 2021, 28, 101431. [CrossRef]

33. Xia, W.F.; Khan, M.I.; Qayyum, S.; Khan, M.I.; Farooq, S. Aspects of constructive/destructive chemical reaction with activation energy for Darcy-Forchheimer hybrid nanofluid flow due to semi-infinite asymmetric channel with absorption and generation features. Ain Shams Eng. J. 2021, 12, 2981-2989. [CrossRef]

34. Aleem, M.; Asjad, M.I.; Shaheen, A.; Khan, I. MHD Influence on different water based nanofluids $\left(\mathrm{TiO}_{2}, \mathrm{Al}_{2} \mathrm{O}_{3}, \mathrm{CuO}\right)$ in porous medium with chemical reaction and Newtonian heating. Chaos Solitons Fractals 2020, 130, 109437. [CrossRef]

35. Sadiq, M.; Hayat, T. Darcy-Forchheimer stretched flow of MHD Maxwell material with heterogeneous and homogeneous reactions. Neural Comput. Appl. 2019, 31, 5857-5864. [CrossRef]

36. Khashi'ie, N.S.; Arifin, N.; Pop, I.; Wahid, N. Flow and heat transfer of hybrid nanofluid over a permeable shrinking cylinder with Joule heating: A comparative analysis. Alex. Eng. J. 2020, 59, 1787-1798. [CrossRef]

37. Jawad, M.; Shah, Z.; Islam, S.; Bonyah, E.; Khan, A. Darcy-Forchheimer flow of MHD nanofluid thin film flow with Joule dissipation and Navier's partial slip. J. Phys. Commun. 2018, 2, 1-17. [CrossRef]

38. Haider, F.; Hayat, T.; Alsaedi, A. Flow of hybrid nanofluid through Darcy-Forchheimer porous space with variable characteristics. Alex. Eng. J. 2021, 60, 3047-3056. [CrossRef]

39. Tiwari, R.K.; Das, M.K. Heat transfer augmentation in a twosidedlid-driven differentially heated square cavity utilizing nanofluids. Int. J. Heat Mass Transf. 2007, 50, 2002-2018. [CrossRef]

40. Devi, S.A.; Devi, S.S. Numerical investigation of hydromagnetic hybrid $\mathrm{Cu}-\mathrm{Al}_{2} \mathrm{O}_{3} /$ water nanofluid flow over a permeable stretching sheet with suction. Int. J. Nonlin. Sci. Numer. Simul. 2016, 17, 249-257. [CrossRef] 
41. Devi, S.S.; Devi, S.A. Numerical investigation of three dimensional hybrid $\mathrm{Cu}-\mathrm{Al}_{2} \mathrm{O}_{3} /$ water nanofluid flow over a stretching sheet with effecting Lorentz force subject to Newtonian heating. Can. J. Phys. 2016, 94, 490-496. [CrossRef]

42. Gorla, R.S.R.; Siddiqa, S.; Mansour, M.A.; Rashad, A.M.; Salah, T. Heat source/sink effects on a hybrid nanofluid-filled porous cavity. J. Thermophys. Heat Transf. 2017, 31. [CrossRef]

43. Lin, Y.H.; Li, B.T.; Zheng, L.C.; Chen, G. Particle shape and radiation effects on Marangoni boundary layer flow and heat transfer of copper-water nanofluid driven by an exponential temperature. Powder Technol. 2016, 301, 379-386. [CrossRef]

44. Waini, I.; Ishak, A.; Pop, I. Unsteady flow and heat transfer past a stretching/shrinking sheet in a hybrid nanofluid. Int. J. Heat Mass Transf. 2019, 136, 288-297. [CrossRef] 